\title{
Wann nützt ein Handbuch? Eine handlungsorientierte, empiri- sche Analyse und ihre Ergebnisse
}

\author{
Dutke, Stephan
}

First published in:

Software-Ergonomie '87: Nützen Informationssysteme dem Benutzer?, S. 398-407, Hrsg.: Prof. Dr. W. Schönpflug und Dipl.-Psych. M. Wittstock, Stuttgart 1987

Münstersches Informations- und Archivsystem multimedialer Inhalte (MIAMI) URN: urn:nbn:de:hbz:6-684194600o6 


\title{
WANN NÜTZT EIN HANDBUCH ? \\ EINE HANDLUNGSORIENTIERTE, EMPIRISCHE ANALYSE \\ UND IHRE ERGEBNISSE
}

Stephan Dutke, Berlin

\begin{abstract}
Es wird eine empirische Untersuchung dargestellt, in der rekonstruiert wurde, wie Benutzer eines Textkommunikationssystems Bedienungsprobleme mit $\mathrm{H}$ ilfe des Benutzerhandbuchs lösen. Die Untersuchungsmethode geht von der Zerlegung möglicher Arbeitsaufgaben in Teilaufgaben aus, deren Erledigung durch Teilhandlungen geschieht. Diese sind Analyseeinheit der Untersuchung und werden als Beschreibungseinheit für die Bedienungsanleitung vorgeschlagen. Typische Handlungsverläufe nach der Benutzung des Handbuchs werden anhand von Wahrscheinlichkeitsgraphen dargestellt. Es werden praktische Konsequenzen für die Gestaltung einer Bedienungsanleitung vorgeschlagen.
\end{abstract}

\section{Fragestellung}

Eine Voraussetzung für die Nützlichkeit jedes EDV-Systems im Arbeitsprozeß ist seine Erlern- und Bedienbarkeit. Der Erwerb von Wissen und die Entwicklung von Fertigkeiten im Umgang mit einem neuen System werden nicht nur durch Unterweisungen, sondern auch durch Bedienungsanleitungen und Handbücher unterstützt (Rupietta, 1982). Häufig sind sie neben Auskunftspersonen, die nur zeitweilig zur Verfügung stehen, sogar die einzigen Hilfsmittel des Lernenden. Hat der Benutzer erste Erfahrungen mit dem system gesammelt, so muß er die Anwendung dieses Wissens auf seine spezifischen Arbeitsaufgaben erlernen, $z$. T. selbst erarbeiten. Dies ist ein Lernstadium, in dem der Bediener in besonderem Maße benutzbarer Informationsquellen bedarf (Helmreich, 1986). Doch auch erfahrene Benutzer können durch veränderte Arbeitsabläufe oder -aufgaben, durch nicht vorhergesehene systemreaktionen oder auch durch eigene Bedienungsfehler in eine situation geraten, die sich von der. Routinebenutzung durch völlig neue Anforderungen unterscheidet. Wenn eigenes Wissen nicht zur Lösung von Bedienungsproblemen ausreicht, wird Hilfe benötigt. Befragungen haben gezeigt, daß Hilfe vorzugsweise bei Kollegen 
oder anderen sachkundigen Personen gesucht wird. Häufig bleibt jedoch nur der Rückgriff auf das Hilfesystem, sofern vorhanden, oder auf das Hanđbuch, die Bedienungsanleitung. Von der Beschaffenheit dieser Einrichtungen hängt oft die Bewältigung einer solchen Problemsituation $a b$. Während es viele Überlegungen $z$ ur verständlichen Gestaltung solcher Hilfsmittel in Bezug auf sprachliche und graphische Merkmale gibt (z. B. Groeben, 1982; Langer u. a., 1974; Grosse \& Mentrup, 1982), sind Untersuchungen, die sich mit der Nutzung und Wirkung solcher Materialien in realen Problemlöseprozessen beschäftigen, seltener. Dabei sollte nicht nur von Interesse sein, ob z. B. eine Erklärung sprachlich verständlich ist, sondern auch, ob ihr Inhalt so beschaffen ist, daß der Benutzer sein Bedienungsproblem lösen kann. Hierzu sind eine verständliche Sprache, Vollständigkeit der Informationen sowie die Übereinstimmung von beschriebenen und tatsächlich erforderlichen Bedienungsschritten notwendige Voraussetzungen; hinreichend sind sie allerdings nicht. Über die rein sprachliche Verständlichkeit hinaus soll die Beschreibung von Bedienungsvorgängen so strukturiert sein, daß sie den Informationsbedürfnissen eines zielgerichtet handelnden Benutzers entgegenkommt und seine Orientierung bezüglich möglicher und notwendiger Handlungsschritte in konkreten Arbeitssituationen unterstützt.

\section{Untersuchungsmethode}

Benutzerinnen eines Textbearbeitungs- und kommunikationssystems (12 Anfängerinnen und 15 erfahrene Arbeitskräfte) erhielten Aufgaben zur Bearbeitung. Das Gerät wird über Funktionstasten und Menüs gesteuert. Vor Versuchsbeginn wurde mit Hilfe von Fragebögen das individuelle Wissen der Versuchspersonen über die Bedienelemente des Geräts und über Handlungsabläufe erhoben. Die Aufgaben waren umgangssprachlich formuliert und enthielten keine expliziten Anweisungen über die Lösungsmethode. Beispiel: "Bitte senden Sie den Mitteilungstext 'A' an Herrn ... in ..., TTX-Rufnummer ...; vergewissern Sie sich, daß der Text oxdnungsgemäß empfangen wurde."

Der Text "A" lag unter diesem Namen gespeichert auf der Diskette vor. Die Bearbeitung der Aufqaben aurch die Versuchsteilneh- 
mer wurde auf Tastendruckniveau automatisch aufgezeichnet. So erhält man freilich nur eine sequentielle Liste von Tastendrukken, aus der der Handlungsverlauf nicht vollständig zu rekonstruieren ist. Eine handlungstheoretische Lösung bietet sich an: die Gliederung dieses scheinbar kontinuierlichen Verhaltensstroms durch die Analyse der Zielstruktur (z. B. V. Cranach u. a., 1980). Deshalb wurde vorher für jede Aufgabe der kürzeste Lösungsweg ermittelt. Diese Lösungswege wurden durch Teilziele gegliedert, die nicht mehr aufgabenspezifisch waren (z. B. Inhaltsverzeichnis der Diskette aufrufen). Jedem der Teilziele wurden nun die entsprechenden operationen (Tasten- und Schalterbedienungen) zugeordnet, die zur Erreichung des betreffenden Teilziels notwendig sind. Somit war der minimale Handlungsraum in folgende hierarchische Komponenten zerlegt: (1) Lösungswege ganzer Aufgaben, (2) Teilhandlungen und (3) Operationen. Im einzelnen wurden 27 verschiedene Tasten- und Schalterbedienungen (Operationen) identifiziert, aus denen 36 unterschiedliche Teilhandlungen generiert werden konnten. Aus diesen 36 Teilhandlungen konnten die Lösungswege aller elf Aufgaben konstruiert werden. Die Aufgaben sind jeweils mit minimal drei und maximal neun Teilhandlungen zu bewältigen, eine Teilhandlung besteht aus wenigstens einer und höchstens 16 Operationen. Die Handlungsabläufe wurden nicht auf der Ebene der Tastendrucke, sondern auf der Ebene von Teilhandlungen analysiert.

Geriet eine Versuchsperson in Schwierigkeiten, stand ihr kein anderes Hilfsmittel als das Handbuch für dieses System zur Verfügung. Trat ein solcher Fall ein, klärte der Versuchsleiter in einem kurzen Dialog, (1) an welcher Aufgabe die Benutzerin gerade arbeitete, (2) an welcher Stelle der Aufgabenbearbeitung sie sich befand, (3) worin ihrer Meinung nach das Problem besteht, (4) welche Informationen sie im Handbuch zu finden hofft und (5) wo sie diese Informationen suchen wird. Die Such- und Lesezeiten wurden registriert, ebenso die Abschnitte der Bedienungsanleitung, in denen die Versuchsperson tatsächlich las. Diese Angaben dienten auch dazu, die entsprechenden Stellen in den Handlungsprotokollen $z u$ identifizieren, so daß für jede Aufgabenbearbeitung angegeben werden konnte, in welcher situation das Handbuch benutzt wurde. Die Versuchsperson konnte, wenn sie der Ansicht war, neue Erkenntnisse gewonnen zu haben, wieder am 
Gerät probieren oder auch erneut das Handbuch zu Rate ziehen. Eine Aufgabe wurde beendet, wenn die Versuchsperson sie nach eigener Einschätzung gelöst hatte, oder aber nach mindestens einem Versuch angab, auch mit Hilfe der Bedienungsanleitung keine Lösung zu finden.

Zur Bewertung der einzelnen Teilhandlungen wurde ein Fehlerkategoriensystem entwickelt, das die zuordnung jeder Teilhandlung in eine der sechs oberkategorien erforderte.

1) Die Teilhandlung wurde fehlerhaft ausgeführt, so daß das Teilziel nicht erreicht wurde (Ausführungsfehler: AF).

2) Die Teilhandlung stellt die erfolgreiche Wiederholung einer zuvor falsch ausgeführten Teilhandlung dar (erfolgreiche wiederholung: EW).

3) Die Teilhandlung wurde richtig ausgefüht. Es handelt sich jedoch um eine Teilhandlung, die den Benutzer an dieser stelle der Aufgabenbearbeitung dem Aufgabenziel nicht näher bringt, sondern zusätzliche negative Konsequenzen verursacht (Verwendungsfehler: VF).

4) Die Teilhandlung wurde richtig ausgeführt und kompensiert negative Effekte einer zuvor falsch verwendeten Teilhandlung (Kompensation: $\mathrm{KO}$ )

5) Die an dieser Stelle des Handlungsverlaufs zur Exreichung des Aufgabenziels notwendige Teilhandlung wurde ersatzlos unterlassen (ausgelassene Teilhandlung: ATH).

6) Die Teilhandlung ist sowohl bezüglich ihrer Ausführung als auch einer zieldienlichen Verwendung fehlerfrei (richtige Teilhandlung: RTH).

Nach der Kodierung der Teilhandlungen wurden aus den Protokollen alle Handlungssequenzen extrahiert, in denen das Handbuch benutzt wurde. Diese wurden so gewählt, daß für jede Handbuchbenutzung die drei unmittelbar vorangegangenen und die drei folgenden Teilhandlungen registriert wurden. Dadurch erhielten alle 307 Handlungssequenzen die gleiche Form: sieben Teilhandlungsschritte, wobei "Benutzung des Handbuchs" immer der mittlere ist. Das Ziel der Datenanalyse bestand darin, Beziehungen zwischen bestimmten Ausgangssituationen und typischen Wirkungen (Handlungsverlauf nach der Benutzung) $\mathrm{zu}$ finden. Hierzu wurde eine Variante der "Lag Sequential Analysis" nach Allison \& Liker (1982) benutzt. Es handelt sich dabei um ein probabili- 
stisches Modell, mit dem die Übergangswahrscheinlichkeiten von einer Handlungsphase zur nächsten bestimmt werden können. Da das Modell die Berechnung éines Varianzterms erlaubt, können diese Wahrscheinlichkeiten auf Signifikanz getestet werden. Dadurch ist es möglich, diejenige Klasse von Handlungsschritten zu bestimmen, die mit nicht zufälliger Häufung als Folge bestimmter anderer Handlungsschritte auftritt.

\section{Ergebnisse und Schlußfolgerungen}

Aus den Ergebnissen der Lag-Analysen können Wahrscheinlichkeitsgraphen konstruiert werden, die die wahrscheinlichsten Handlungssequenzen abbilden. Die Darstellungen haben ihren Ausgangspunkt im Ereignis "Bedienungsanleitung lesen" ( Lag $_{O}=$ BDA). Lag $_{1}$ bis $\operatorname{Lag}_{3}$ bezeichnen die drei nachfolgenden Teilhandlungen. Durchgezogene Linien kennzeichnen eine gegenüber der Basiswahrscheinlichkeit der jeweiligen Teilhandlungsklasse erhöhte Auftretenswahrscheinlichkeit (mit *: signifikant erhöht, p<.05); gestrichelte Linien bezeichnen gegenüber der Basiswahrscheinlichkeit verminderte Auftretenswahrscheinlichkeiten (mit * : signifikant vermindert).

Abbildung 1 zeigt die zwei wahrscheinlichsten Teilhandlungssequenzen im Falle einer erfolglosen Benutzung des Handbuchs. Als "erfolglos" wird eine Teilhandlungssequenz bezeichnet, bei der die dritte Teilhandlung nach der Handbuchbenutzung noch nicht die erforderliche, richtig ausgeführte Teilhandlung ist. Die Rate der erfolgreichen Handbuchbenutzungen liegt nach dieser Definition zwischen $14 \%$ und $57 \%$, ohne daß eine eindeutige Abhängigkeit von der Exfahrung der Versuchspersonen festzustellen wäre.

Der erste Weg stellt einen wiederholten Wechsel zwischen erforderlicher, aber falsch ausgefuhrter Teilhandlung und vergeblichem Nachschlagen in der Anleitung dar (BDA-AF-BDA-AF). Der zweite Weg besteht aus Teilhandlungen, die an dieser stelle der Aufgabenbearbeitung nicht zielführend sind, und aus einem Versuch der Kompensation der negativen Folgen dieser Verwendungsfehler in $\mathrm{Lag}_{3}(\mathrm{VF}-\mathrm{VF}-\mathrm{KO})$. Der úbergang von Lago zu Lag, ist jedoch im Gegensatz zum ersten Weg nicht signifikant. Dies bedeutet, daß die Auftretenswahrscheinlichkeit der Kette VF-Vf-KO nach Benutzung des Handbuchs nicht wahrscheinlicher ist 
als an jeder anderen Stelle der Aufgabenbearbeitung. Die Wahrscheinlichkeit, daß nach der Handbuchbenutzung die erforderliche Teilhandlung ohne weitere Versuche richtig ausgeführt wird, ist mit .03 signifikant gegenüber der Basiswahrscheinlichkeit vermindert (BDA-RTH). Die Utbergangswahrscheinlichkeiten zwischen den zwei Wegen (AF-VF, BDA-RO, VF-BDA und VF-AF) sind alle vermindert, in drei Fällen signifikant. Dies spricht für eine $\mathrm{Ab}-$ hängigkeit der Teilhandlungsklassen innerhalb der Wege bzw. für eine hohe Unabhängigkeit der Wege voneinander.

Abb. 1 Erfolglose Benutzung des Handbuchs

$$
\mathrm{LAG}_{\mathrm{O}} \quad \mathrm{LAG}_{1} \quad \mathrm{LAG}_{2} \quad \mathrm{LAG}_{3}
$$

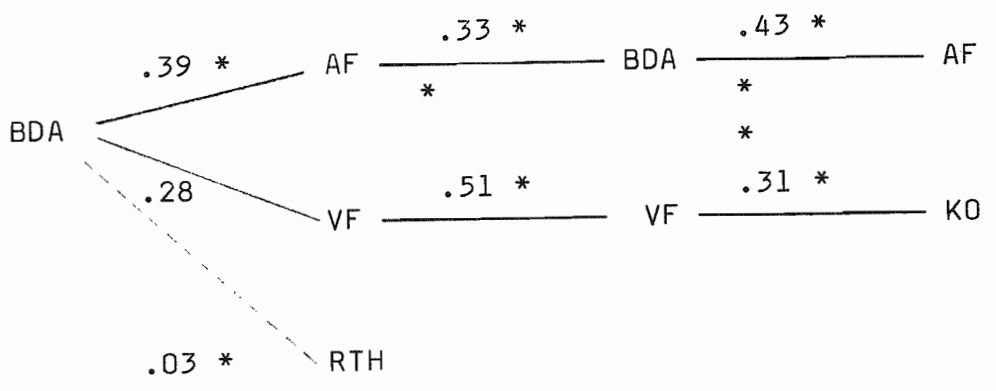

Abbildung 2 zeigt die erfolglosen Teilhandlungssequenzen für die Fälle, in denen die Versuchsperson in der Anleitung nach einer anderen als der an dieser Stelle der Aufgabenbearbeitung erforderlichen Teilhandlung suchte. Der Weg wiederholter Ausführungsfehler tritt nicht mehr in Erscheinung, da die Versuchspersonen in diesen Fällen offenbar nicht wissen, welche Teilhandlung sie dem Aufgabenziel näher bringt. Die Sequenz der wiederholten Verwendungsfehler $(\mathrm{VF}-\mathrm{VF}-\mathrm{VF})$ ist intern signifikant miteinander verknüpft, doch der übergang von Lago $\mathrm{zu} \mathrm{Lag}_{1}$ (BDA-VF) ist wiederum nicht signifikant wahrscheinlicher als zu anderen Zeitpunkten des Handlungsverlaufs. Kompensatorische (BDA-KO) und richtige Teilhandlungen (BDA-RTH) folgen in diesen Fällen signifikant seltener einer Benutzung der Bedienungsanleitung. Signifikant erhöht ist die Wahrscheinlichkeit eines Aufgabenabbruchs $(B D A-A B B)$.

Die erfolgreich verlaufenen Teilhandlungssequenzen sind 
Abb. 2 Erfolglose Benutzung des Handbuchs: Suchen nach einer anderen als der erforderlichen Teilhandlung
$L A G_{0}$
$L A G_{1}$
$L A G_{2}$
$\mathrm{LAG}_{3}$

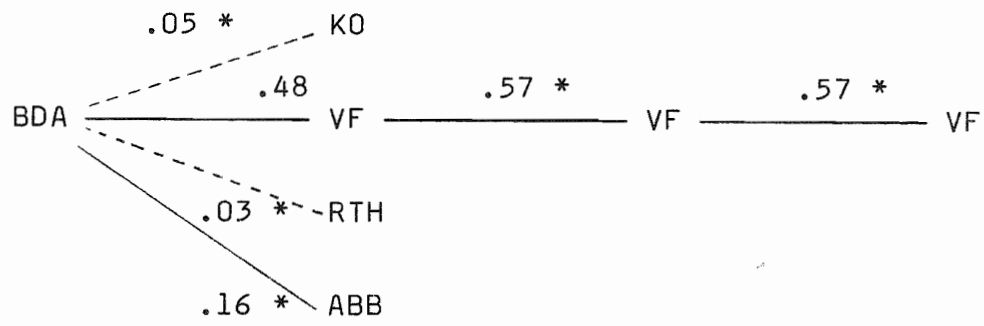

Abb. 3 Erfolgreiche Benutzung des Handbuchs
$L A G_{0}$
$L A G_{1}$
$L A G_{2}$
$\mathrm{LAG}_{3}$

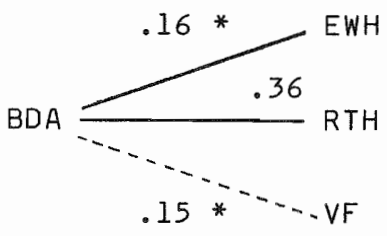

in Abbildung 3 dargestellt. Der Anteil der Teilhandlungen, die ohne vorherige Fehler in Lag, richtig ausgefüht wurden, beträgt zwar mehr als ein Drittel aller in dieser phase registrierten Teilhandlungen, doch ist die wahrscheinlichkeit hierfür nicht signifikant erhöht. Die Steigerung der erfolgreichen wiederholung und die Verminderung der Verwendungsfehler nach der Lektüre der Bedienungsanleitung sind jedoch signifikant.

Zusammenfassend sind folgende Tendenzen festzustellen:

1) Falsch ausgeführte Teilhandlungen können nach mehrmaligem Probieren und Nachschlagen in der Anleitung häufig nicht korrigiert werden. Die versuchspersonen verharren dennoch häufig in der strategie, das ziel mit der gleichen Teilhandlung durch wiederholte versuche doch noch zu erreichen. Ein Strategiewechsel, 
$z$. B. durch andere Teilhandlungen oder "Umwege" das gleiche Teilziel zu erreichen, ist recht unwahrscheinlich und wird durch die Lektüre der Bedienungsanleitung nicht gefördert, eher behindert.

2) Weiß die Benutzerin nicht, mit Hilfe welcher Teilhandlung sie das nächste Teilziel bzw. das Aufgabenziel erreichen kann, ist die Wahrscheinlichkeit, in der Anleitung eine Lösung zu finden, äußerst gering. Der größte Teil der Aufgabenabbrüche durch die Versuchsteilnehmer geschieht in diesen situationen.

3) Die erfolgreiche Benutzung des Handbuchs spiegelt sich lediglich in der erhöhten Wahrscheinlichkeit für erfolgreiche Wiederholungen vorher falsch ausgeführter Teilhandlungen wider. Dies bedeutet, daß die Benutzer bereits vor der Lektüre der Bedienungsanleitung wußten, welches Teilziel mit welcher Teilhandlung als nächste anzustreben sei. Falsch eingesetzte oder kompensatorische Teilhandlungen, die ein Indiz für das Umgehen des Problems auf alternative oder auch umständlichere Weise sein könnten, sind nach der Handbuchbenutzung signifikant seltener als es ihrer Basiswahrscheinlichkeit entspräche.

Eine Analyse der Mißerfolgsfälle ergab, daß die Beschreibung im Handbuch oft an den einzelnen Programmfunktionen (z. B. Einfügen, Löschen) orientiert ist. Für den Benutzer erweist es sich jedoch als sinnvoller, diese Abläufe als Bestandteil einer komplexen Arbeitshandlung darzustellen. Unter dieser Perspektive sollten die Beschreibungseinheiten Teilhandlungen sein, die aus der Zielanalyse möglicher Arbeitsaufgaben gewonnen werden können. 'Die vollständige Beschreibung einer Teilhandlung sollte folgende Komponenten umfassen:

1) Beschreibung der Zweckbestimmungen dieser Teilhandlung.

2) Beschreibung der Bedingungen, unter denen diese Teilhandlung erfolgreich ausgeführt werden kann.

3) Beschreibung der Abfolge von Bedienungsschritten, mit denen das angegebene Teilziel erreicht werden kann.

4) Beschreibung der Konsequenzen dieser Teilhandlung für den Systemzustand und den zustand des Arbeitsobjekts bei fehlerfreier Ausführung.

In vielen Fällen werden die Bestandteile 1, 2 und 4 gegenüber der Abfolge der tatsächlichen Bedienungsschritte vernachlässigt. Werden die möglichen Zweckbestimmungen nicht voll- 
ständig erläutert oder an nachgeordneter Position beschrieben, kann es geschehen, daß der Benutzer nicht erkennt, welche Teilhandlung in einer gegebenen Handlungssituation zweckdienlich ist. In vielen Fällen wurden Teilhandlungen, obwohl die Benutzer die Ausführung nachweislich beherrschten, nicht zur Problemlösung eingesetzt, weil sie nicht um den instrumentellen Wert dieser Prozeduren wußten.

Werden die Ausführungsbedingungen nicht vollständig und vor allem vor der Darstellung des Handlungsablaufs beschrieben, können Mißerfolge eintreten, deren Ursachen für den Benutzer nur schwer zu erkennen sind. Häufig wurde immer wieder die ausgeführte und die in der Anleitung beschriebene Abfolge der Bedienungsschritte verglichen und korrekt umgesetzt. Trotzdem mißlang die Teilhandlung, und das ziel wurde nicht erreicht, weil eine notwendige, aber nicht explizit erwähnte Bedingung nicht erfüllt war.

Die Konsequenz einer korrekt ausgeführten. Teilhandlung besteht nicht nur aus der Realisierung des ziels, sondern häufig auch aus einer Anzahl weiterer Effekte auf den Systemzustand und den Zustand des Arbeitsobjekts. Werden sie nicht vollständig beschrieben, besteht die Gefahr, daß der Benutzer bei der planung nachfolgender Handlungsschritte von falschen Voraussetzungen ausgeht. Dies kann zum MiBlingen späterer Teilhandlungen führen. Eine an den obigen vier Gestaltungsregeln orientierte Beschreibung von Bedienungsabläufen beugt Schwierigkeiten und Mißerfolgen vor.

\section{Literatur}

Allison, P. D. \& Liker, J. D. (1982): Analyzing sequential data on dyadic interaction: a comment on Gottman. Psychological Bulletin, 91 (1982), 293-403.

Cranach, V. M., Kalbermatten, U., Indermühler, K., \& Gugler, B. (1980): Zielgerichtetes Handeln. Bern: Huber.

Groeben, N. (1982): Leserpsychologie. Münster: Aschendorff. Grosse, S., \& Mentrup, W. (Hrsg.). (1982): Anweisungstexte. Forschungsberichte des Instituts für Deutsche Sprache, Mannheim, Band 54. Tübingen: Narr.

Helmreich, R. (1986): Planning user acceptance - a management task. In proceedings part II of the International Scientific 
Conference: Work with Display Units. Stockholm, May 12-15, 1986 (pp. 777-780).

Langer, I., Schulz V. Thun, F., \& Tausch, R. (1974): Verständlichkeit in Schule, Verwaltung und Wissenschaft. München: Reinhardt.

Rupietta, W. (1982): Dokumentation als Information über die Benutzung von Computersystemen. Angewandte Informatik, 11 $(1982), 536-540$.

Diese Forschungsarbeiten wurden finanziell und technisch durch die Firma Siemens, Erlangen und München, unterstützt.

Dipl.-Psych. Stephan Dutke Institut für Psychologie Freie Universität Berlin Habelschwerdter Allee 45 1000 Berlin 33 